\title{
DATA COMMUNICATIONS OVER AIRCRAFT POWER LINES
}

\author{
Hai Tian, Tom Trojak \\ Teletronics Technology Corporation
}

Charles Jones PhD

Edwards Air Force Base

\begin{abstract}
This paper introduces a study of the feasibility and initial hardware design for transmitting data over aircraft power lines. The intent of this design is to significantly reduce the wiring in the aircraft instrumentation system. The potential usages of this technology include Common Airborne Instrumentation System (CAIS) or clock distribution. Aircraft power lines channel characteristics are presented and Orthogonal Frequency Division Multiplexing (OFDM) is introduced as an attractive modulation scheme for high-speed power line transmission. A design of a full-duplex transceiver with accurate frequency planning is then discussed. A general discussion of what communications protocols are appropriate for this technology is also provided.
\end{abstract}

\section{KEYWORDS}

Flight Test Instrumentation, Airborne Instrumentation, High-Speed Data Transmissions, Power Line Communications, OFDM, Full Duplex, FDD

\section{INTRODUCTION}

Current instrumentation systems are dependent on installing wires throughout a test vehicle independent of existing wires on the vehicle. The ability to transfer data over power lines is a technology that continues to mature. Power line networks already exist throughout most aircraft vehicles. It thus seems appropriate to consider implementing data over power lines for test and evaluation since this could reduce installation costs and schedules significantly. The results in this paper are from a project specifically targeting data over power line for use with the Common Airborne Instrumentation System (CAIS) and even more specifically for insertion into airframe, propulsion, and avionics (APA) test and evaluation programs. However, these results can be used to evaluate the practicality of this technology for a larger range of communication protocols. For this type of evaluation, there are several areas of technical concern to look at: simplex vs. duplex, time criticality, and maximum bit rate.

The first area of concern is simply whether communication is one-way (simplex) or two-way (duplex). The duplex case might be further broken down into the question of whether the two 
communications must be at the same time or not. Ethernet, in some sense, is multiplex in that any two nodes can talk to each other. However, Ethernet's contention-based protocol only allows one node to transmit at a time and thus Ethernet is ultimately a half-duplex protocol. Examples of simplex include distributing time or transmission of a data cycle map (often referred to as a PCM matrix). CAIS, on the other hand, is a hard full-duplex protocol that requires commands and responses to be transmitted simultaneously. This is implemented by having separate command and response wires in conventional CAISs.

The second area of concern, time criticality, relates to whether or not data must be at a certain point within strict time limitations. Ethernet is not time critical in that packets can be resent or routed any number of ways; the packets don't even have to arrive in sequence since they can be rearranged upon arrival. CAIS, on the other hand, is very time critical. A response must arrive with a time delay equal to four command words after the command is sent, with a leeway of about 600 nanoseconds roundtrip. This leeway exists because of the nondeterministic delay across different lengths of wire (roughly 1.7 to 2.0 nanoseconds per foot).

Maximum bit rate, the third area, is a concern not only from the point of view of how much data can be transmitted but also from a protocol point of view. The internet relays data over different wires that work at different bit rates without a problem. However, each direction of CAIS requires $5 \mathrm{Mbps}$ because of the time critical, command and response, nature of the protocol.

The characteristics of the desired physical layer - the aircraft power line - are the driving forces in the current application. As will be discussed, these characteristics lead to the choice of orthogonal frequency division multiplexing (OFDM) for modulation. Understanding both the characteristics of the physical medium and OFDM then allows us to discuss what protocols have practical applications on the power line.

\section{AIRCRAFT POWER LINE CHARACTERISTICS}

Power lines were not designed as a broadband data transmission medium. They present a harsh environment for high-frequency signals. Three critical factors of interest are: noise, channel transfer function (attenuation), and impedance.

According to [1], there are five categories of noise in power lines:

1) Colored background noise with a relatively low power spectral density (PSD). This type of time-varying noise can be seen as a summation of all low power noise sources.

2) Narrowband noise consisting of amplitude modulated sinusoidal signals, which is caused by broadcast stations.

3) Periodic impulsive noise synchronous to the main's frequency with multiple rates.

4) Periodic impulsive noise asynchronous to the main's frequency caused by switching of rectifier diodes of power supplies. 
5) Asynchronous impulsive noise caused by switching transients in the network. This is the most harmful noise for data transmission. Its duration varies and the PSD of the noise may reach $50 \mathrm{~dB}$ above the background noise.

The Channel Transfer Function is determined by many factors. Multipath is a primary corruptor of an ideal channel. Multipath due to reflection results in multiple echoes of a signal arriving at a receiver at different times. In a power distribution system, reflections result from lack of termination, poorly defined line impedance, and the existence of line stubs. Multiple echoes of the same signal arrive at the receiver at different times. The time spreading in a certain symbol causes intersymbol interference (ISI), which affects the subsequent arriving symbol. As a result, the bit error rate (BER) is increased. In the frequency domain, multipath brings frequency-selective fading, which randomly affects only a portion of the overall bandwidth at any certain time. The notches can be very deep and are unpredictably located. Both the multipath effect and the frequency-selective fading can vary with time.

Impedance of the power line is determined by devices plugged into the line and the number of branches. The time-varying impedance of the power line creates a challenge for the transceiver design. Since the impedance of the transceiver has to be fixed, an impedance mismatch between the transceiver and the power line occurs.

In this project, $+28 \mathrm{~V}$ DC power lines, as defined in MIL-STD-704 [6], are used as the transmission medium. An initial test of aircraft $+28 \mathrm{Vdc}$ power line channel characteristics was performed at Edwards AFB, California. The transmission band between $10 \mathrm{MHz}$ and $100 \mathrm{MHz}$ in the $+28 \mathrm{Vdc}$ power lines were tested. Three test points were used in this test (Figure 1 red circles). Two links were tested (Figure 1 blue dot lines): Test Link 1 was from the left wing to the right wing, and Test Link 2 was from the left wing to under the cockpit on the right side.

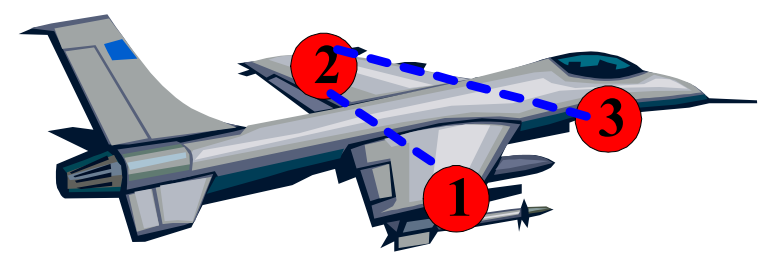

\section{Figure 1 Test Points and Test Links}

The electrical power distribution system is a bus structure. Powered devices are grouped by function (weapon, navigation, etc.) and each group can be connected and disconnected by a single switch on a circuit breaker. Wires carry all the electrical power to devices. There are fuse and distribution boxes in the aircraft's central section. Each powered device on the aircraft has its own 'hot' wire connected to the central boxes. The device's power returns (or grounds) are through the aircraft's chassis or frame. This approach with a chassis ground is similar to a car.

This wired bus structure makes high-speed data transmission difficult for at least two reasons.

1. A data transmission path between two devices will see many various-length dangling stubs. Every other device on that particular bus is wired to the single circuit breaker 
controlling that bus. It is from that common point that power to all other devices on that bus originates. This is the origin of all the dangling stubs for that bus. Even at moderate RF frequencies, each stub will look like a short circuit to ground at some frequency. This causes frequency-selective fading. In Figure 2 and Figure 3, each notch in the transfer function is a short circuit to ground from a stub.

2. Since there is no return line, the characteristic impedance of a single line is poorly controlled. If the return had a dedicated wire and it ran in proximity to the power line, characteristic impedance would be at least mildly controlled.

The forward transfer functions from 10 to $100 \mathrm{MHz}$ of Test Link 1 and Test Link 2 are shown in Figure 2 and Figure 3 . The low pass channel characteristic can be observed in both figures. The premium transmission channel is the frequency band below $20 \mathrm{MHz}$. The frequency band from $20 \mathrm{MHz}$ to $60 \mathrm{MHz}$ has the medium attenuation. High attenuation is observed at the frequency band higher than $60 \mathrm{MHz}$.

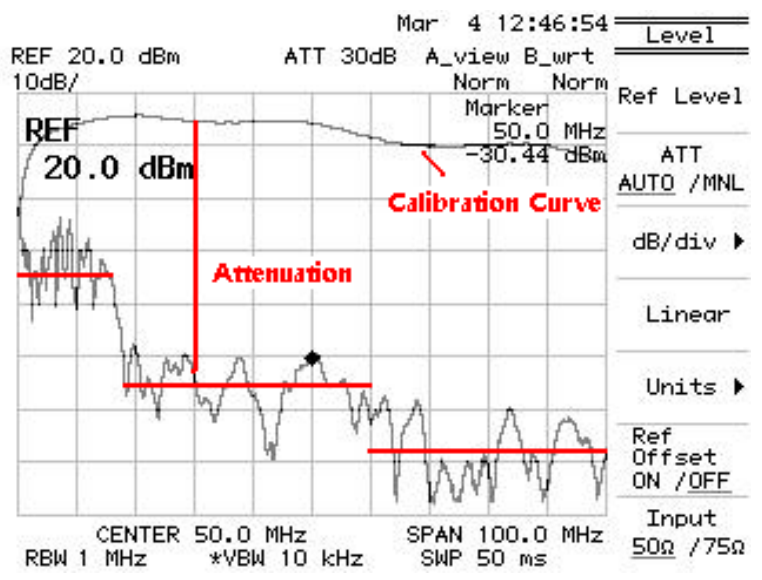

Figure 2 Forward Transfer Function of Test Link 1 from Left Wing to Right Wing (10dB/Division)

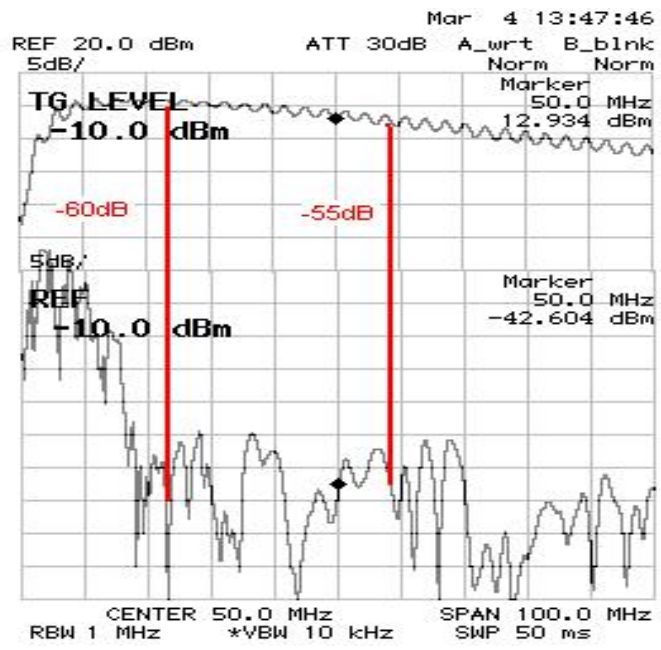

Figure 3 Forward Transfer Function of Test Link 2 from Left Wing to Right Side Under Cockpit (5dB/Division) 
Frequency-selective fading was also observed during the test. The fading creates the deep notches, which are randomly located over the entire frequency band. Some examples are shown in Figure 4, which also shows that some notches have $30 \mathrm{~dB}$ more attenuation than the average value.
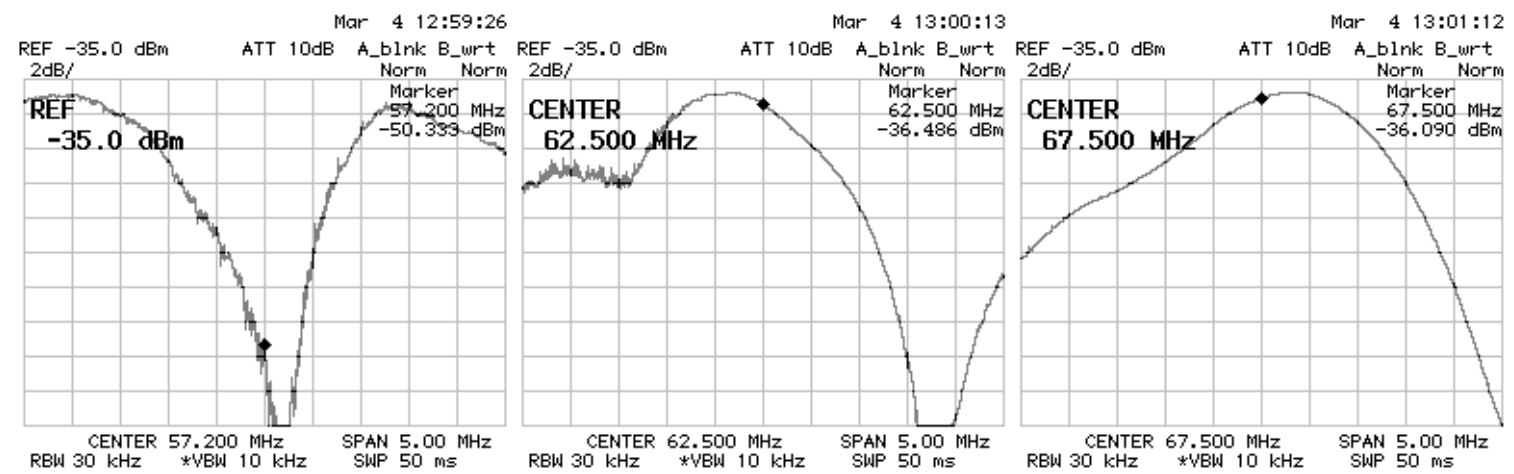

Figure 4 Example of Frequency Selective Fading on Test Link 1

As in the earlier discussion, time-varying impedance of power line precludes a fixed equalization approach. This lack of equalization results in an impedance mismatch and poor return loss. A large portion of injected power is reflected back towards the signal source. This effect is shown in Figure 5 as both high attenuation and notches.

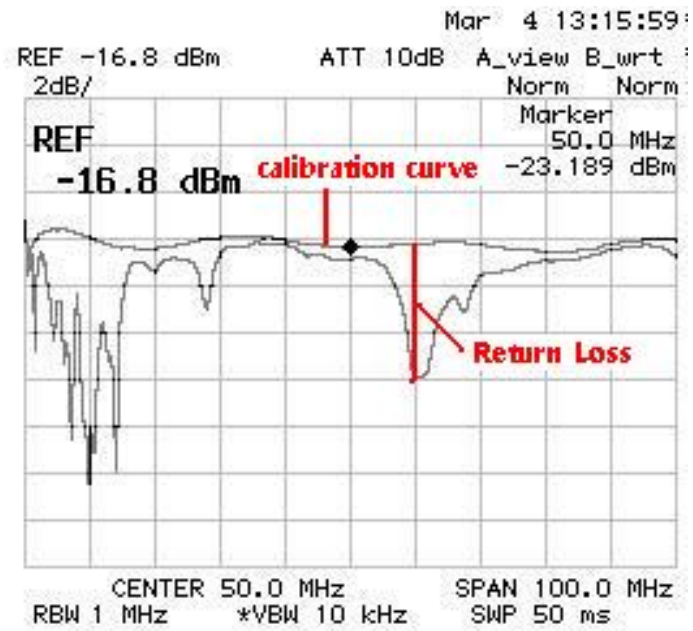

Figure 5 Input Return Loss at Left Wing Test Point

\section{ORTHOGONAL FREQUENCY DIVISION MULTIPLEXING (OFDM)}

The OFDM is a multicarrier modulation scheme where a transmission band is subdivided into a number of independent subchannels. These subchannels are carefully spaced such that their frequencies are orthogonal to each other. Figure 6 shows such a signal spectrum. Each subcarrier is located on all the other subchannels' spectra zero crossing points. By sampling at the subchannel's center frequency, spectral orthogonality is maintained. 


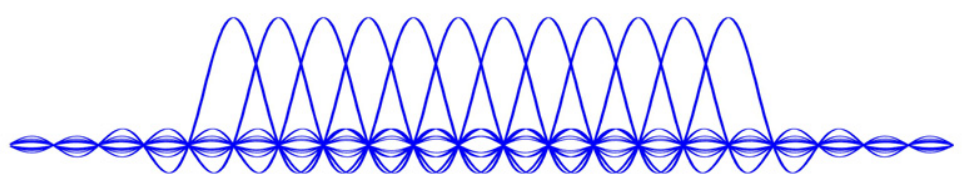

Figure 6 OFDM Signal

The OFDM offers several advantages over alternative methods. For instance, in the traditional frequency division multiplexing (FDM) used in cable TV and analog radio broadcast, the aggregate bandwidth must be divided into numerous subchannels, with guard bands spaced between those subchannels to reduce interference. OFDM makes better use of the aggregate bandwidth; due to the inherent orthogonality of subchannels, OFDM requires no guard bands. The available bandwidth can be 'packed' more densely, allowing the spectrum to be used more efficiently.

The OFDM modulation proves to be very robust in overcoming multipath delay spread. By spreading the OFDM symbol across a number of subchannels, the symbol period for each subchannel can be lengthened significantly, resulting in reduced ISI. Furthermore, by introducing a guard interval into the symbol that is larger that the expected delay spread, ISI can be practically eliminated. Typically, the contents of the guard interval are made to be a "cyclic prefix' of data repeated from the end of the symbol period. Consequently, an acquisition time window the length of a symbol period can vary in position by as much as the guard interval and still successfully recover the entire symbol without intersymbol interference. This cyclic prefix can also be beneficially used for timing recovery and synchronization in the receiver.

Since the data rate in each subchannel is relatively low compared to single carrier schemes, each subchannel has a relatively flat passband frequency response, reducing the need for equalization. The relative narrowness in frequency of each subchannel also helps minimize ripple in the passband of a given subchannel.

The OFDM is robust against time domain impulsive noise, frequency domain narrowband interference, and frequency selective fading. While an impulsive noise occupies a wide bandwidth that can affect a number of subchannels, its time duration is very short. The long symbol duration that exists in OFDM transmission tends to minimize the effect of this short impulsive noise. OFDM is robust against narrowband interference because this type of interference only affects a small percentage of channels. An automatic adaptive algorithm is used in the OFDM transceiver to turn off those subchannels with a low signal-to-noise ratio. Forward error correction (FEC) techniques can help further alleviate this form of interference. The OFDM is not without its disadvantages. It is more sensitive to frequency offset and phase noise, and has a relatively large peak-to-average power ratio. However, these disadvantages tend to be outweighed by the advantages of OFDM in relevant applications.

Figure 7 shows a block diagram of the OFDM transmitter section as implemented in this project in a field-programmable gate array (FPGA). 


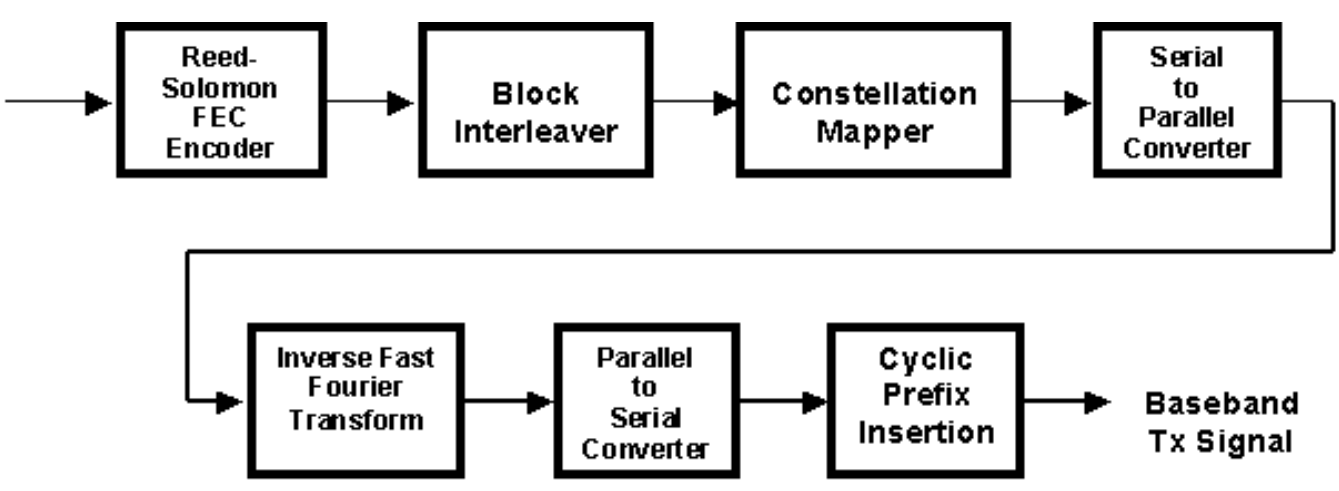

Figure 7 OFDM Transmitter Block Diagram

The incoming data stream first enters the Reed-Solomon Forward Error Correction (RS-FEC) Encoder block. RS-FEC is a symbol oriented error correction coding technique that, in addition to its excellent ability to correct random errors, provides superior burst error correcting capability. Data transmission performance is greatly improved under adverse conditions found in the power line environment using this technique. After encoding, data is passed to the Block Interleaver, which helps reduce burst error rates within the data channel by spreading out 'clumps' of errors over a larger region. Data next passes into the Constellation Mapper where symbols are mapped to appropriate constellation points according to the modulation scheme of choice, which in turn results in In-phase (I) and Quadrature (Q) signal generation. After serial to parallel conversion, the $\mathrm{I}$ and $\mathrm{Q}$ data streams are fed to a 128-point Inverse Fast Fourier Transform (IFFT) block, where each of 128 subchannels gets modulated onto its own specific orthogonal subcarrier. After a parallel to serial conversion, a Cyclic Prefix (CP) is inserted into the guard interval at the beginning of the OFDM symbol, and the resulting baseband signal gets passed on to the transmitter up converter.

The OFDM receiver section does essentially the inverse of the transmitter section. Baseband signals from the down converter section have the Cyclic Prefix removed, converted to parallel data and sent to a Fast Fourier Transform (FFT) block for demodulation into the appropriate subchannels. After Demapping and Deinterleaving, the result is sent to the RS-FEC Decoder, where transmission errors are detected and corrected as per the RS algorithm.

\section{FREQUENCY PLANNING AND TRANSCEIVER STRUCTURE}

To achieve full duplex communications, frequency division duplex (FDD) is adopted in this project. High-speed data can be transmitted and received in two separate frequency bands (downlink and uplink) simultaneously without collision. There are two types of transceiver: the Master Transceiver (MT) transmits signals through downlink and receives signals through uplink. The Slave Transceiver (ST) transmits signals through uplink and receives signals through downlink. Both types of transceivers have the same structure, which is shown in Figure 8.

Careful frequency planning must be accomplished since the available frequency band is limited $(<100 \mathrm{MHz})$ and the signal bandwidth is relatively broad. The receiving path frequency planning of ST is given in Figure 9 as an example. 


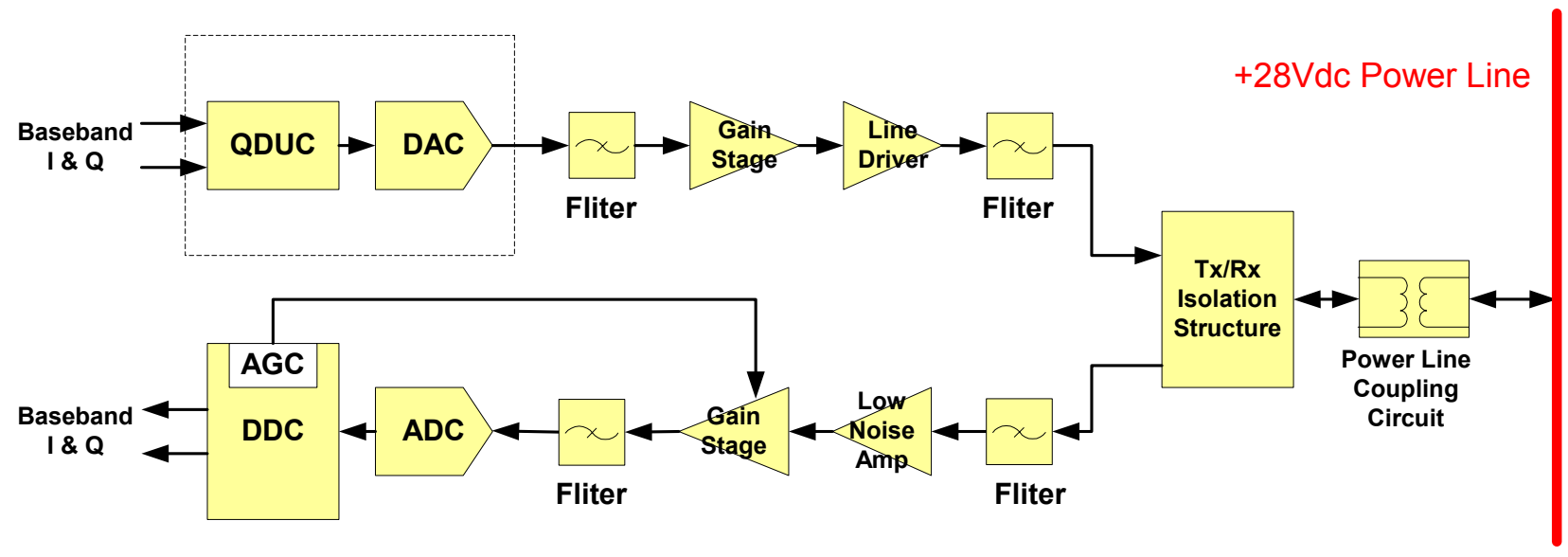

Figure 8 Power Line Transmitter Block Diagram

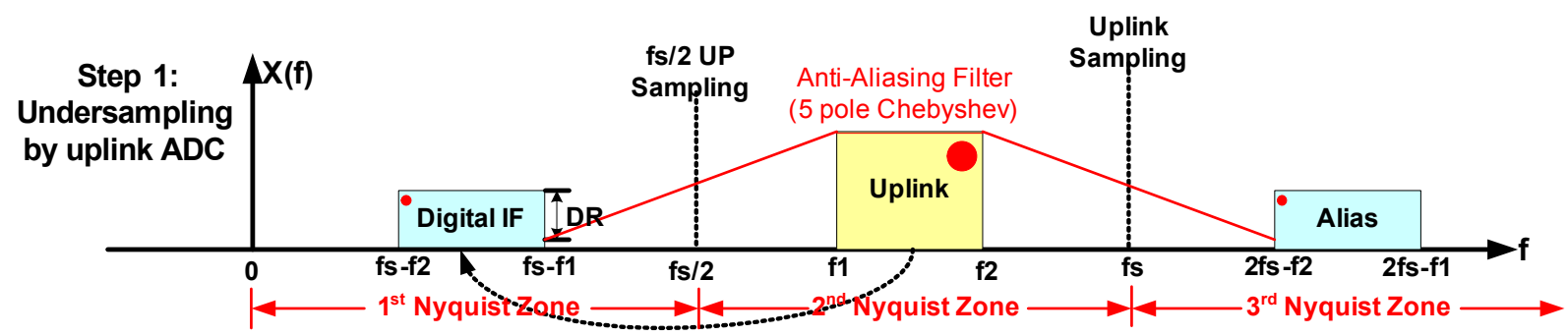

Step 2:

\begin{tabular}{c|c}
$\begin{array}{c}\text { Quadrature } \\
\text { Demodulation } \\
\text { by uplink DDC }\end{array}$ & Digital Filter
\end{tabular}

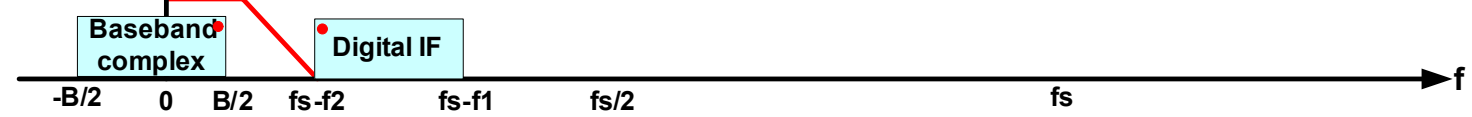

Figure 9 Frequency Planning of ST's Rx path

Based on conventional baseband sampling theory, the sampling rate $\left(f_{s}\right)$ of the analog-to-digital converter (ADC) must be higher than 2 times the highest frequency component of interest $\left(f_{2}\right)$, that is $f_{s}>2 f_{2}$. In actual practice, at least 3 times the sampling rate is required to relax the antialiasing filter requirements. For instance, if $f_{2}=75 \mathrm{MHz}$, we need a high-speed ADC operating at $225 \mathrm{MHz}$ to perform sampling.

Instead of baseband sampling, undersampling can be used here to reduce ADC sampling rates. According to the Nyquist criteria described in [4]: a signal that has frequency components between $f_{1}$ and $f_{2}$ must be sampled at a rate $f_{s}>2\left(f_{2}-f_{1}\right)$ in order to preserve all the signal information. In other words, the sampling rate only needs to be higher than twice the information bandwidth regardless of the location of the information band. For example, if no anti-aliasing filter is used before the sampling, signals in all the higher order Nyquist zones will translate down into the first Nyquist zone (DC to $f_{2} / 2$ ). All aliases are overlapping and cannot be 
distinguished. If a band pass filter (BPF) is applied to the information band located in a higher order Nyquist zone, all signals in the other Nyquist zones will be filtered out (including the frequency components in the first Nyquist zone). After ADC sampling, the information band will translate down into the first Nyquist zone as an alias. Since no other frequency components exist (filtered out by BPF before sampling), this alias can be easily distinguished. It contains all the information in the original information band and nothing else. The undersampling results in only the original information band being down-converted into the first Nyquist zone. One more thing needs to be mentioned here: undersampling results in a frequency reversal for those frequency components located in even order Nyquist zones, hence a frequency reordering needs to be completed in the post processing.

In this transceiver design, a $15 \mathrm{MHz}$ bandwidth signal is located from $60 \mathrm{MHz}\left(f_{1}\right)$ to $75 \mathrm{MHz}$ $\left(f_{2}\right)$ and $90 \mathrm{MHz}\left(f_{s}\right)$ is chosen as the sampling rate of the ADC. Hence, the signal is located in the second Nyquist zone (shown in Figure 9). A 5-pole Chebyshev BPF is used as the anti-aliasing filter. After undersampling, a digital IF band is obtained between $15 \mathrm{MHz}\left(f_{s}-f_{2}\right)$ and $30 \mathrm{MHz}\left(f_{s}-f_{1}\right)$. The red dots at the corner of these signals indicate the frequency reversal.

The digital IF band needs to be placed carefully to avoid overlapping between the fundamental frequency and harmonics [5]. In this transceiver design, the fundamental frequency of the digital IF signal is placed between $15 \mathrm{MHz}$ and $30 \mathrm{MHz}$. The second harmonic is located between $30 \mathrm{MHz}$ and $60 \mathrm{MHz}$, which is out-of-band. A digital down converter (DDC) demodulates the digital IF signal to the baseband quadrature I and Q signals. The frequency reordering is done in the DDC. Numerically controlled oscillators (NCO) in the DDC are tuned to $66.5 \mathrm{MHz}$ (an alias of $-22.5 \mathrm{MHz}$ ) instead of $22.5 \mathrm{MHz}$. After decimation and digital filtering, I and Q signals are fed into the OFDM receiver for further processing.

\section{DISCUSSION}

An overriding concern of transmitting data over an aircraft power line is safety. This manifests itself in two ways: internal and external to the power line. From a data point of view, we are concerned with the interference from power lines destroying bits, but it is also possible for the data signal to interfere with other devices connected to the power lines. Externally, since the power cables are not shielded, there is a real potential of causing electromagnetic interference with other devices on the vehicle. Thus, although there are many factors involved, when all trade-offs are analyzed, this environment limits bit rate since you are limited in both transmission frequencies and power.

The physical environment provides strong justification for the use of OFDM. However, as discussed, in order to implement OFDM there is some necessary processing. Initial estimates of processing time using an FPGA going one way is on the order of a microsecond. A round trip of data must go through four translations so that round trip processing is on the order of several microseconds. (Contrast this with the minimum command response time of approximately 10 microseconds in CAIS.) 
The CAIS bus operates at 10 Mbps utilizing bi-phase-space (BI $\varphi$-S) encoding. The processing time introduces some difficulties with daisy-chaining multiple CAIS devices. Each device must know fairly precisely when to transmit a response and the introduced delays do not allow for this level of precision. Thus, implementing a strict CAIS protocol probably limits the use of the power line to one link in the CAIS bus. Although it may be possible to implement more CAIS bus links over the power line, the protocol used would have to appear to be CAIS to the devices but would distinctly not be CAIS on the power lines. The conclusion is thus that CAIS is not the best candidate for this technology. However, there are still applications for the level of CAIS that, when implemented over power lines, would achieve significant cost and schedule savings.

Understanding the limitations of data over power lines technology allows us to identify protocols that match the technology. Perhaps the overriding limit is the processing time necessary to convert a signal into OFDM. This means that time-critical protocols are not well suited for this. The maximum bit rate is of concern as well. However, if the protocol is not time critical, then this is less of a concern; data is just slowed down to whatever bit rate is available just as it is on the Internet. The only real concern is whether or not you have more data than can fit through the pipe in an acceptable time. These limitations leave a variety of protocols and applications for which data over power line is practical. Distribution of time is practical, although extreme precision may require system-specific analysis or implementation of adaptive techniques. Transmission of discretes as used in device command and control is practical as is data cycle map transmission or any truly one way communication. More complex protocols that are not time critical could also be implemented. This includes protocols such as Ethernet or MIL-STD 1553 [7].

\section{ACKNOWLEDGEMENT}

This effort is being funded by the Test Insertion and Risk Reduction (TIRR) program under contract number F04700-02-D-0005.

\section{REFERENCES}

[1] Götz, M., "Power Line Channel Characteristics and Their Effect on Communication System Design", IEEE Communications Magazine, vol. 42, no. 4, Apr., 2004, pp. 78-86.

[2] Hanzo, L., "Introduction to Orthogonal Frequency Division Multiplexing", OFDM and MC-CDMA for Broadband Multi-User Communications, WLANs and Broadcasting, IEEE Press and John Wiley, Chichester, West Sussex, England, 2003, pp. 23-72.

[3] Meyr, H., "Baseband Communications", Digital Communication Receivers, John Wiley, New York, NY, 1998, pp. 61-206.

[4] Analog Devices technical Staff, "Analog-Digital Conversion", Fundamentals of Sampled Data Systems, Analog Devices Application Notes.

[5] Brannon, B., "Basics of Designing a Digital Radio Receiver (Radio 101)", Analog Devices Application Notes, pp.3.

[6] MIL-STD 704F, Department of Defense Interface Standard, Aircraft Electric Power Characteristics, 12 March 2004.

[7] MIL-STD 1553b, Military Standard, Aircraft Internal Time Division Command/Response Multiplex Data Bus, Jan. 1996. 